NORDITA $92 / 45$

July, 1992

\title{
Superstring One-Loop and Gravitino Contributions to Planckian Scattering
}

\author{
Alessandro Bellini* \\ NORDITA, Blegdamsvej 17, DK-2100 Copenhagen Ø, Denmark \\ and INFN, Sezione di Firenze, Largo E. Fermi 2, I-50125 Firenze, Italy \\ Marco Ademollo and Marcello Ciafaloni \\ Dipartimento di Fisica dell'Università, Firenze and INFN, Sezione di Firenze \\ Largo E. Fermi 2, I-50125 Firenze, Italy
}

\begin{abstract}
Corrections to the semiclassical approximation in nearly forward Planckian energy collisions are here reconsidered. Starting from the one-loop superstring amplitude, we are able to disentangle the first subleading high-energy contribution at large impact parameters, and we thus directly compute the one-loop correction to the superstring eikonal. By comparing this result with previous ones by Amati, Ciafaloni and Veneziano (ACV) for pure gravity, we identify one-loop gravitino contributions which agree with previous results by Lipatov. We finally argue, on the basis of analyticity and unitarity, that gravitinos do not contribute at all to the large distance two-loop ACV correction, which thus acquires a universal "classical" interpretation.
\end{abstract}

\footnotetext{
* Supported in part by A. Della Riccia Foundation.
} 


\section{Introduction}

Gravitational scattering at Planckian energies and small angles was investigated in the past [1-4] and is still under discussion $[5,6]$, in order to understand the role of short distances in string theory and quantum gravity. A similar investigation was also performed [7] from the point of view of reggeized graviton exchange in the multi-Regge kinematics.

The high energy regime $2 E=\sqrt{s}>m_{\text {Planck }}$ is characterized [3] by a strong effective coupling $\alpha_{G}=G s / \hbar$, so that a resummation of the perturbative series is needed. Various approaches have shown that the leading contributions to the scattering amplitude at a given impact parameter $b$ yields a semiclassical, eikonal approximation

$$
\begin{aligned}
S(b, E) & =e^{2 i \delta_{0}(b, E)}, \\
\delta_{0} & =-\frac{G s}{\hbar} \log b
\end{aligned}
$$

where the unobservable (IR singular) Coulomb phase has been omitted.

Corrections to the leading result (1.1) involve additional powers of $G$, occurring in the dimensionless combinations

$$
\begin{aligned}
\frac{\hbar G}{b^{2}} & =\frac{\lambda_{P}^{2}}{b^{2}}, \quad\left(\lambda_{P}^{2} \equiv G \hbar\right) \\
\frac{G^{2} s}{b^{2}} & =\frac{R^{2}}{b^{2}}=\alpha_{G} \frac{\lambda_{P}^{2}}{b^{2}},
\end{aligned}
$$

and, for the string case [3], also in the combination

$$
\frac{G \hbar}{b^{2}} \frac{\alpha^{\prime} \hbar}{b^{2}}=\frac{\lambda_{P}^{2}}{b^{2}} \frac{\lambda_{s}^{2}}{b^{2}}, \quad\left(\lambda_{s}^{2} \equiv \alpha^{\prime} \hbar\right) .
$$

They are small for sufficiently large values of $b$ with respect to the Planck length $\lambda_{P}$, the gravitational radius $R=2 G E$, and, in the string case, also the string length $\lambda_{s}$. Since $R$ is a classical parameter, we refer to the corrections of type (1.2b) as classical ones, while we refer to those of type in eq. (1.2a) (eq. (1.3)) as quantum (string) corrections.

No complete theory of such corrections exists so far. However, a large class of string corrections (1.3) and the first perturbative terms of type (1.2) were computed in ACV I [3] and ACV II [4]. In the $\alpha^{\prime} \rightarrow 0$ limit their result can be expressed in terms of an effective eikonal representation for the elastic scattering amplitude

$$
S(b, E)=\exp 2 i\left(\delta_{0}+\delta_{1}+\delta_{2}\right),
$$


where, for pure gravity,

$$
\delta_{1}=\frac{6}{\pi} \frac{G^{2} s}{b^{2}} \log s, \quad \quad \operatorname{Re} \delta_{2}=2 \frac{G^{3} s^{2}}{\hbar b^{2}} .
$$

The result (1.5) involve precisely the parameters $\delta_{1} / \delta_{0} \simeq \lambda_{P}^{2} / b^{2}$ and $\delta_{2} / \delta_{0} \simeq R^{2} / b^{2}$ of eqs. (1.2), and were obtained in ref. [4] by computing $\operatorname{Im} \delta_{2}$ directly, and using an analyticity argument in order to obtain $\delta_{1}$ and $\operatorname{Re} \delta_{2}$ for the case of pure gravity.

In this paper we reconsider the calculation of $\delta_{1}$ in superstring theory, by a direct evaluation of the high energy behavior of the one-loop string amplitude.

Since the one-loop leading behavior was already analyzed in the past $[3,8]$ and is embodied in eq. (1.1) through the $O\left(G^{2}\right)$ term $i \delta_{0}^{2}$, the real problem is to disentangle the first subleading contribution. Previous attempts [8] have failed, due to the difficulty of properly subtracting the leading term.

From the point of view of graviton Regge pole exchange, the relevant contributions to the amplitude in momentum transfer $\mathbf{q}$ space

$$
\frac{1}{s} A\left(s, \mathbf{q}^{2}\right)=4 \int d^{2} \mathbf{b} e^{i \mathbf{b} \cdot \mathbf{q}} \frac{e^{2 i \delta(\mathbf{b}, E)}}{2 i}
$$

are the following:

(1) At tree level, corresponding to single Regge pole exchange ${ }^{\star}$, we obtain

$$
\frac{1}{s} \mathrm{~A}^{(0)}\left(s, \mathbf{q}^{2}\right) \simeq 4 \tilde{\delta}_{0}=\frac{8 \pi G s}{\mathbf{q}^{2}}
$$

(2) At one loop level we have similarly

$$
\frac{1}{4 s} \mathrm{~A}^{(1)}\left(s, \mathbf{q}^{2}\right)=i \tilde{\delta}_{0}^{2}+\tilde{\delta}_{1}
$$

where $\tilde{\delta}_{n} \equiv \tilde{\delta}_{n}(\mathbf{q}, E)$ indicate the Fourier transforms of $\delta_{n}(\mathbf{b}, E)$. In particular

$$
i \tilde{\delta}_{0}^{2} \simeq i \frac{2 \pi G^{2} s^{2}}{\mathbf{q}^{2}} \log \mathbf{q}^{2}
$$

corresponds to the leading Regge cut, while

$$
\tilde{\delta}_{1} \simeq-6 G^{2} s^{2} \log s \log \mathbf{q}^{2}
$$

\footnotetext{
* We omit the shrinkage terms in the $\alpha^{\prime} \rightarrow 0$ limit.
} 
is the Regge pole renormalization. The latter was already computed, on the basis of a generalized soft graviton emission current $[7,9]$ in the model of reggeized graviton exchange of ref. [7].

In order to disentangle the subleading contribution (1.10) in string theory, we start considering the representation of the one-loop superstring amplitude over the torus and we identify the region in moduli space which is relevant for the pole renormalization (sect. 2). This region overlaps with the cut region, and a careful subtraction is needed in order to obtain a finite expression (sect. 3).

Our final result (sect. 4) has the same form as the one in eq. (1.5) for pure gravity, but contains additional contributions that we ascribe to gravitinos, as expected in SST theory, leading to $\mathrm{N}=8$ supergravity in the field theory limit. This interpretation agrees with Lipatov's [7] calculation of the gravitino contributions.

\section{High energy behavior of superstring one-loop amplitude}

The large $s$ and fixed $t$ asymptotic limit of the graviton-graviton scattering amplitude was discussed in ACV I for the case of Type II superstring theory to one loop. In that paper it was shown that the leading contribution is given by the exchange of two gravireggeon poles, corresponding to a Regge cut in the angular momentum $J$ plane. We give in the following an expression containing the subleading term, which is proved to be a double pole in the $J$-plane, according to the general expectation of graviton Reggeization.

In general we expect quantum and string corrections to contribute to this double pole at fixed $t$, but in $D=4$ and in the limit $\alpha_{c}^{\prime} \mathbf{q}^{2} \rightarrow 0$ we shall show that only quantum corrections of type (1.2a) survive. In principle this could be directly achieved by Feynman graph computation in $N=8$ supergravity, the low energy quantum field theory derived from Type II superstring with toroidal compactification of six dimensions. However, the string calculation already contains the UV-cutoff $\alpha_{c}^{\prime}$, and turns out to be simpler. 
The superstring scattering amplitude, in units $\alpha_{c}^{\prime}=\frac{1}{2} \alpha^{\prime}=\hbar=c=1$, is given by

$$
\begin{aligned}
A_{4}=-2 g_{D}^{2} K_{c l} & \left\{\frac{\Gamma\left(-\frac{1}{2} s\right) \Gamma\left(-\frac{1}{2} u\right) \Gamma\left(-\frac{1}{2} t\right)}{\Gamma\left(1+\frac{1}{2} s\right) \Gamma\left(1+\frac{1}{2} u\right) \Gamma\left(1+\frac{1}{2} t\right)}\right. \\
& \left.-g_{10} \frac{1}{2\left(16 \pi^{3}\right)^{2}} \int_{F} \frac{d^{2} \tau}{(\operatorname{Im} \tau)^{5}} F_{2}\left(\tau, R_{c}\right) \int \prod_{r=a, b, c} d^{2} \nu_{r} \prod_{r<s} \chi_{r s}^{2 k_{r} k_{s}}\right\}
\end{aligned}
$$

where $K_{c l}$ is the standard superstring kinematical factor whose asymptotic behavior is

$$
K_{c l} \sim\left(\frac{s}{2}\right)^{4} \varepsilon_{a} \cdot \varepsilon_{d} \varepsilon_{b} \cdot \varepsilon_{c}
$$

The integral in moduli space is over the fundamental region $F$ of the torus topology [10], while $F_{2}$ is the toroidal compactification factor for closed string. Finally $\chi_{r s}=$ $\chi\left(e^{2 \pi i\left(\nu_{r}-\nu_{s}\right)}, e^{2 \pi i \tau}\right)$ is given in terms of the Jacobi $\Theta$ function and its asymptotic behavior $(\tau \rightarrow i \infty, \nu / \tau>0$ fixed $)$ relevant for our calculation is

$$
\chi_{r s}=\chi\left(e^{2 \pi i\left(\nu_{r}-\nu_{s}\right)}, e^{2 \pi i \tau}\right)=\text { const. } \times \exp \left[-\pi \frac{(\operatorname{Im} \nu)^{2}}{\operatorname{Im} \tau}+\operatorname{Re}\left(i \pi \nu+e^{2 \pi i \nu}+e^{2 \pi i(\tau-\nu)}\right)\right]
$$

The $s$ and $t$ dependence in the integrand of (2.1) appears in the factor

$$
f=\left(\frac{\chi_{a d} \chi_{b c}}{\chi_{a c} \chi_{b d}}\right)^{-t}\left(\frac{\chi_{a b} \chi_{c d}}{\chi_{a c} \chi_{b d}}\right)^{-s}
$$

By using for the $\chi$ 's the asymptotic form (2.2) we obtain

$$
\frac{\chi_{a d} \chi_{b c}}{\chi_{a c} \chi_{b d}}=\left|4 \sin \pi\left(\nu_{d}-\nu_{a}\right) \sin \pi\left(\nu_{b}-\nu_{c}\right) e^{-2 \pi x(1-x) \tau_{2}}\right|
$$

where we have set $x=\operatorname{Im}\left(\nu_{b}+\nu_{c}\right) / 2 \tau_{2}, \quad \tau_{2}=\operatorname{Im} \tau$, and

$$
\begin{aligned}
\frac{\chi_{a b} \chi_{c d}}{\chi_{a c} \chi_{b d}} & =\exp \left\{\frac{2 \pi}{\tau_{2}} \operatorname{Im}\left(\nu_{a}-\nu_{d}\right) \operatorname{Im}\left(\nu_{b}-\nu_{c}\right)\right. \\
& \left.-\operatorname{Re}\left[8 \sin \pi\left(\nu_{d}-\nu_{a}\right) \sin \pi\left(\nu_{b}-\nu_{c}\right) e^{i \pi \tau} \cos \pi\left(\tau-\nu_{d}-\nu_{a}+\nu_{b}+\nu_{c}\right)\right]\right\} .
\end{aligned}
$$

In the limit $s \rightarrow i \infty$ there are two regions that can contribute: one is the cut region discussed in ACV I

$$
\operatorname{Im}\left(\nu_{a}-\nu_{d}\right) \operatorname{Im}\left(\nu_{b}-\nu_{c}\right) \sim O\left(s^{-1}\right), \quad \tau_{2} \gtrsim O(\log s)
$$


the other is related to the pinching configuration of fig. 1, i.e.

$$
\left|\nu_{d}-\nu_{a}\right|\left|\nu_{b}-\nu_{c}\right| \sim O\left(s^{-1}\right), \quad \text { any } \tau_{2} \text {. }
$$

The latter is the double pole region we are interested in, to which in general all values of $\tau_{2}$ contribute. However, in the region $\mathbf{q}^{2} \rightarrow 0$ relevant for large values of $b$, the dominant behavior will come from large values of $\tau_{2}$. This explains a posteriori why the large $\tau_{2}$ behavior (2.2) is relevant, but also shows that cut and pole regions do overlap, which makes it difficult to extract the double pole correction.

In order to perform the cut subtraction we first recast eq.(2.1) in a more manegeable form, valid in both regions (2.5a) and (2.5b), for all values of the phases of $\left(\nu_{d}-\nu_{a}\right)$ and $\left(\nu_{b}-\nu_{c}\right)$. Following ACV we introduce the variables $a, b, c$ and perform the integrations over $\operatorname{Re} \tau$ and $a$, which provide each a Bessel function. This is still possible also in the region $(2.5 \mathrm{~b})$ because the last term in the exponent of $(2.4 \mathrm{~b})$ can be written as:

$$
\begin{aligned}
\ldots & +4 \rho_{+} \rho_{-} \exp \left[-2 \pi \tau_{2} x-\pi \operatorname{Im}\left(\nu_{d}-\nu_{a}\right)\right] \cos \phi_{1} \\
& +4 \rho_{+} \rho_{-} \exp \left[-2 \pi \tau_{2}(1-x)+\pi \operatorname{Im}\left(\nu_{d}-\nu_{a}\right)\right] \cos \phi_{2}
\end{aligned}
$$

where we have set $\nu_{d}=\tau$, we have introduced the shorthand notation

$$
\rho_{+}=\left|\sin \pi\left(\nu_{d}-\nu_{a}\right)\right|, \quad \rho_{-}=\left|\sin \pi\left(\nu_{b}-\nu_{c}\right)\right|
$$

and the two phases $\phi_{1,2}$ are linear in $\tau_{1}$ and $a$ and defined by

$$
\phi_{1}=2 \pi \tau_{1}+2 \alpha_{b c}-\phi_{2}, \quad \phi_{2}=2 \pi a+\alpha_{b c}+\alpha_{a d},
$$

where e.g. $\alpha_{b c}$ is

$$
\alpha_{b c}=\arcsin \frac{2 \sinh \pi \operatorname{Im}\left(\nu_{b}-\nu_{c}\right) \cos \pi \operatorname{Re}\left(\nu_{b}-\nu_{c}\right)}{\left[2 \cosh 2 \pi \operatorname{Im}\left(\nu_{b}-\nu_{c}\right)-2 \cos 2 \pi \operatorname{Re}\left(\nu_{b}-\nu_{c}\right)\right]^{1 / 2}} .
$$

Finally, neglecting $\operatorname{Im}\left(\nu_{d}-\nu_{a}\right)$, with respect to $\tau_{2} x$ in the exponents of eq.(2.6), we obtain the following expression, which contains the Regge cut and the double pole singularity:

$$
A_{4}=(8 \pi G)^{2}\left(\frac{\sigma}{2}\right)^{3} I, \quad s=i \sigma, \quad \epsilon=\frac{4-D}{2}
$$




$$
\begin{aligned}
I= & \int_{0}^{1} d x \int_{0}^{\infty} d \tau_{2}\left(8 \pi^{2} \tau_{2}\right)^{\epsilon} \int_{0}^{\tau_{2}} d \operatorname{Im}\left(\nu_{d}-\nu_{a}\right) \int_{-\tau_{2}}^{+\tau_{2}} d \operatorname{Im}\left(\nu_{b}-\nu_{c}\right) \\
& \times \frac{2 \sigma}{\tau_{2}} \exp \left[i \frac{2 \pi \sigma}{\tau_{2}} \operatorname{Im}\left(\nu_{d}-\nu_{a}\right) \operatorname{Im}\left(\nu_{b}-\nu_{c}\right)\right] \int_{-1 / 2}^{+1 / 2} d \operatorname{Re}\left(\nu_{d}-\nu_{a}\right) \\
& \times \int_{-1 / 2}^{+1 / 2} d \operatorname{Re}\left(\nu_{b}-\nu_{c}\right)\left(4 \rho_{+} \rho_{-}\right)^{q^{2}} \exp \left[-2 \pi \tau_{2} q^{2} x(1-x)\right] \\
& \times J_{0}\left(4 \sigma \rho_{+} \rho_{-} e^{-2 \pi \tau_{2} x}\right) J_{0}\left(4 \sigma \rho_{+} \rho_{-} e^{-2 \pi \tau_{2}(1-x)}\right)
\end{aligned}
$$

The Regge cut dominates for Ret $<2$. Such a dominant contribution has to be carefully subtracted before we can calculate the trajectory renormalization.

\section{Cut subtraction and pole renormalization}

We now proceed to integrate the external leg insertions on two small circular regions $\left|\nu_{d}-\nu_{a}\right|<c, \quad\left|\nu_{b}-\nu_{c}\right|<c$ around the points $\nu_{d}=\nu_{a}$ and $\nu_{b}=\nu_{c}$. This pinching region may be singled out alternatively, by studying the asymptotic behavior of the integral expression in eq. (2.1) for $s \rightarrow i \infty$ with the method of stationary phase, as first pointed out by Sundborg [8]. At the points above the phase is stationary in $\tau_{2}$ and $x$, and the argument of the exponential in eq. (2.4b) is small, implying the absence of non-Regge exponential dependence on $s$.

To do the integral, we introduce polar coordinates in both circular regions, we perform the angular integrations by using the representation

$$
\int_{0}^{2 \pi} \frac{d \theta_{1} d \theta_{2}}{4 \pi^{2}} \exp \left(i 2 z \sin \theta_{1} \sin \theta_{2}\right)=J_{0}^{2}(z)
$$

and we obtain the result

$$
\begin{aligned}
I= & \int_{0}^{1} d x \int_{0}^{\infty} d \tau_{2}\left(8 \pi^{2} \tau_{2}\right)^{\epsilon} e^{-2 \pi \tau_{2} q^{2} x(1-x)} \int d \rho_{+} d \rho_{-}\left(4 \rho_{+} \rho_{-}\right)^{q^{2}} \frac{4 \sigma \rho_{+} \rho_{-}}{\pi^{2} \tau_{2}} \\
& \times J_{0}^{2}\left(\frac{\sigma \rho_{+} \rho_{-}}{\pi \tau_{2}}\right) J_{0}\left(4 \sigma \rho_{+} \rho_{-} e^{-2 \pi \tau_{2} x}\right) J_{0}\left(4 \sigma \rho_{+} \rho_{-} e^{-2 \pi \tau_{2}(1-x)}\right)
\end{aligned}
$$

We now expand the two last $J_{0}$ functions in power series of their arguments and we replace the sums with Sommerfeld-Watson transforms in the $n, m$ complex planes 
obtaining:

$$
\begin{aligned}
I= & \frac{(4 \pi)^{\epsilon}}{2 \pi^{3}} \int_{\gamma} \frac{d n}{2 \pi i} \frac{d m}{2 \pi i} \frac{\Gamma(-n) \Gamma(-m)}{\Gamma(n+1) \Gamma(m+1)}\left(\frac{\sigma}{2}\right)^{2 n+2 m} \int_{0}^{1} d x \int_{0}^{\infty} d \tau_{2} \tau_{2}{ }^{\epsilon} e^{-B \tau_{2}} \\
& \times \int_{0}^{c} d \rho_{-} d \rho_{+}\left(\rho_{-} \rho_{+}\right)^{2 n+2 m+q^{2}}\left[\left(\frac{\sigma}{\tau_{2}} \rho_{-} \rho_{+} J_{0}^{2}\left(\frac{\sigma}{\pi \tau_{2}} \rho_{-} \rho_{+}\right)-1\right)+1\right] .
\end{aligned}
$$

where we have defined

$$
B=\mathbf{q}^{2} x(1-x)+2 n x+2 m(1-x)
$$

It is worthwhile to discuss more the expression (3.2a). We expect that the magnitude of the two circular regions, in the pinched geometry of fig. 1, is not relevant to asymptotics. This is certainly true for the integral of the terms in round brackets, which is convergent for large $\sigma$ due to the property

$$
\sigma p J_{0}^{2}\left(\frac{\sigma}{\pi} p\right) \underset{\sigma \rightarrow \infty}{\longrightarrow} 1+\sin \left(2 \frac{\sigma}{\pi} p\right)
$$

but not for the last term in square brackets, which produces a factor $\sim(c \sigma)^{2 n+2 m}$, to be interpreted as the tail of the cut contribution in the pole region. By subtracting the last term we obtain the pole contribution

$$
\begin{aligned}
I_{\text {pole }}= & \frac{(4 \pi)^{\epsilon}}{2 \pi^{3}}\left(\frac{\sigma}{2}\right)^{-1-\mathbf{q}^{2}} \log \sigma \int_{\gamma} \frac{d n}{2 \pi i} \frac{d m}{2 \pi i} \frac{\Gamma(-n) \Gamma(-m)}{\Gamma(n+1) \Gamma(m+1)} \\
& \times \int_{0}^{1} d x \int_{0}^{\infty} d \tau_{2} \tau_{2}{ }^{\epsilon} e^{-B \tau_{2}} \int_{0}^{\infty} d z z^{A}\left[\frac{2 z}{\tau_{2}} J_{0}^{2}\left(\frac{2 z}{\pi \tau_{2}}\right)-1\right]
\end{aligned}
$$

where

$$
A=2 n+2 m+\mathbf{q}^{2}
$$

and we have kept only the term proportional to $\log \sigma$, to be interpreted as a trajectory renormalization (double pole in the J-plane). The integral with respect to $z$ in the above expression is well defined for $A>-1$, and in particular in the region $A \sim 0$ we are interested in, as a result of the cut subtraction. We evaluate this integral using an alternative integral representation of the same analytic function valid for $-2<A<-1$,

$$
\int_{0}^{\infty} d z z^{A+1} J_{0}^{2}\left(\frac{z}{\pi}\right)=\left(\frac{1}{2 \pi}\right)^{-A-1} \pi \frac{\Gamma(-A-1) \Gamma\left(1+\frac{1}{2} A\right)}{\Gamma\left(-\frac{1}{2} A\right)^{3}}
$$


so we have finally ${ }^{\star}$

$$
\begin{aligned}
I_{\text {pole }}= & \frac{(4)^{\epsilon}}{2 \pi^{4}}\left(\frac{\sigma}{2}\right)^{-1-\mathbf{q}^{2}} \log \sigma \int_{\gamma} \frac{d n}{2 \pi i} \frac{d m}{2 \pi i} \frac{\Gamma(-n) \Gamma(-m)}{\Gamma(n+1) \Gamma(m+1)} \\
& \times \int_{0}^{1} d x\left(\frac{\pi}{B}\right)^{\epsilon+A+2} \Gamma(\epsilon+A+2) \pi \frac{\Gamma(-A-1) \Gamma\left(1+\frac{1}{2} A\right)}{\Gamma^{3}\left(-\frac{1}{2} A\right)} .
\end{aligned}
$$

The $m$ and $n$ integrations of eq. (3.4) are performed in detail in Appendix A in the $\alpha_{c}^{\prime} \mathbf{q}^{2} \rightarrow 0$ limit, by replacing the integration in $x$ with a momentum integral in transverse space as in ACV I. Here we only notice that the $\mathbf{q}^{2}=0$ singularities in (3.4) come from $B=0$ (cfr. eq. (3.2b)) and thus from the large $\tau_{2}$ region, as mentioned before.

The final result, coming from eq. (3.4) and Appendix A, yields the double pole amplitude

$$
\frac{1}{s} \mathrm{~A}_{\text {pole }}^{(1)}\left(s, \mathbf{q}^{2}\right)=\frac{8 \pi G}{\mathbf{q}^{2}} \log s \delta \alpha\left(\mathbf{q}^{2}\right) s^{1-\mathbf{q}^{2}},
$$

corresponding to the J-plane projection

$$
A_{J}^{(1)}=\frac{1}{\left(J-2+\mathbf{q}^{2}\right)^{2}} \frac{8 \pi G}{\mathbf{q}^{2}} \delta \alpha\left(\mathbf{q}^{2}\right)
$$

where $\delta \alpha$ is the trajectory renormalization (for small $\alpha_{c}^{\prime} \mathbf{q}^{2}$ )

$$
\begin{gathered}
\delta \alpha=G \mathbf{q}^{2} \int \frac{d^{2} k}{(2 \pi)^{2}}\left[\frac{\left(\mathbf{q}^{2}-t_{1}-t_{2}\right)^{2}}{t_{1}^{2} t_{2}}+2 \frac{\mathbf{q}^{2}-t_{1}-t_{2}}{t_{1} t_{2}}+(1 \leftrightarrow 2)\right], \\
t_{1}=\mathbf{k}^{2}, \quad t_{2}=(\mathbf{q}-\mathbf{k})^{2} .
\end{gathered}
$$

Note that the singular $\mathbf{q}^{2} \rightarrow 0$ behavior of this expression is not modified by the following rewriting

$$
\begin{aligned}
\delta \alpha=8 \pi G \frac{\mathbf{q}^{2}}{(2 \pi)^{3}} & \int \frac{d^{2} \mathbf{k}}{\mathbf{k}^{2}(\mathbf{q}-\mathbf{k})^{2}}\left[[\mathbf{k} \cdot(\mathbf{q}-\mathbf{k})]^{2}\left(\frac{1}{\mathbf{k}^{2}}+\frac{1}{(\mathbf{q}-\mathbf{k})^{2}}\right)\right. \\
& \left.+4 \mathbf{k} \cdot(\mathbf{q}-\mathbf{k})-\mathbf{q}^{2}\right]
\end{aligned}
$$

which coincides with the Lipatov result [7] for $\mathrm{N}=8$ supergravity.

* The singularity at $A=-1$ of the pole expression (3.4) is actually spurious, because it cancels out with the cut term in the complete expression (3.2). 


\section{One loop correction to the eikonal and gravitino contributions}

The first subleading contribution to the one-loop string amplitude obtained in eq. (3.5) is to be compared with the eikonal representation (1.6). Since the leading Regge cut corresponds to the $i \tilde{\delta}_{0}^{2}$ term in eq. (1.8), the result (3.5) yields directly the expression for $\delta_{1}^{s}$, i.e., the superstring one loop correction to the eikonal

$$
\delta_{1}^{s}(b, s)=\frac{2}{\pi} G \log s \int d^{2} \mathbf{q} \frac{\delta \alpha\left(\mathbf{q}^{2}\right)}{\mathbf{q}^{2}} s^{1-\alpha^{\prime} \mathbf{q}^{2}} e^{i \mathbf{b} \cdot \mathbf{q}}
$$

where, by eq. (3.7a) and (3.8), the singular $\mathbf{q}^{2}$-dependence of $\delta \alpha$ is of the form

$$
\frac{\delta \alpha}{\mathbf{q}^{2}} \simeq-\frac{G}{\pi} \log \frac{\mathbf{q}^{2}}{\lambda^{2}}-2 \frac{G}{\pi} \log \frac{\Lambda}{\mathbf{q}^{2}},
$$

$\lambda(\Lambda)$ being IR (UV) cut-off. By replacing (4.2) into (4.1), we obtain in the $\alpha^{\prime} \rightarrow 0$ limit,

$$
\delta_{1}^{s}(b, s)=-\frac{2}{\pi} \frac{G^{2} s}{b^{2}} \log s .
$$

This expression differs (by a factor of $(-3)$ ) from the pure gravity result (1.5). It is natural to ascribe the above discrepancy to gravitino contributions, that were computed by Lipatov [7] to be

$$
\frac{\delta \alpha_{N}}{\mathbf{q}^{2}}=N G \int \frac{d^{2} k}{4 \pi^{2}} \frac{\mathbf{q}^{2}-t_{1}-t_{2}}{t_{1} t_{2}}
$$

where $\mathrm{N}$ is the number of gravitinos. We see that the integrand in (4.4) is IR finite, but yields an UV contribution

$$
\frac{\delta \alpha_{N}}{\mathbf{q}^{2}}=-\frac{N}{2} \frac{G}{\pi} \log \frac{\Lambda^{2}}{\mathbf{q}^{2}} .
$$

By summing graviton and gravitino contributions we obtain in general

$$
\frac{\delta \alpha}{\mathbf{q}^{2}}=-\frac{G}{\pi} \log \frac{\mathbf{q}^{2}}{\lambda^{2}}+2 \frac{G}{\pi} \log \frac{\Lambda^{2}}{\mathbf{q}^{2}}-\frac{N}{2} \frac{G}{\pi} \log \frac{\Lambda^{2}}{\mathbf{q}^{2}},
$$

which for $N=0$ agrees with eq. (1.5) and for $N=8$ yields the SST result (4.2).

A more direct way of arriving at the result (4.5) is to compute the gravitino contribution to the one-loop absorptive part (fig. 2). By using the graviton-gravitino amplitudes of ref. [12], we find (Appendix B)

$$
\operatorname{Im} \frac{A_{g}\left(s, \mathbf{q}^{2}\right)}{s}=N \pi G^{2} s \log \frac{\Lambda^{2}}{\mathbf{q}^{2}} P_{\uparrow \downarrow}=4 N \int d^{2} \mathbf{b} \operatorname{Im} \delta_{1 g}(s, b) e^{i \mathbf{b} \cdot \mathbf{q}}
$$


where $P_{\uparrow \downarrow}$ denotes the projection operator on s-channel states of opposite helicities. It follows, by using the analyticity arguments of ACV II, that the gravitino contributions to the one-loop eikonal are given by

$$
\delta_{1}^{(N)} \equiv N \delta_{1 g}=\frac{N G^{2} s}{\mathbf{b}^{2}}\left(i P_{\uparrow \downarrow}-\frac{1}{\pi} \log s\right), \quad \frac{\delta \alpha_{N}}{\mathbf{q}^{2}}=-\frac{N}{2} \frac{G}{\pi} \log \frac{\Lambda^{2}}{\mathbf{q}^{2}},
$$

in agreement with eq. (4.5).

We can now discuss the gravitino contribution to the full scattering matrix at Planckian energies. We look for a unitary S-matrix of eikonal type, with the factorized form

$$
S=S^{(G)} S^{(N)}
$$

where the graviton contribution

$$
S^{(G)}=\exp 2 i\left(\delta_{0}+\delta_{1}+\delta_{2}\right) \times(\text { inelastic terms })
$$

is the one found in ACV II and reported in eq. (1.4), while the gravitino contribution is set to be the matrix

$$
S^{(N)}=e^{2 i \delta_{1}^{(N)}}\left(1+2 i \sqrt{\operatorname{Im} \delta_{1}^{(N)}} \sigma_{1} P_{\uparrow \downarrow}\right)
$$

Here $\delta_{1}^{(N)}$ is taken from eq. (4.8) and $\sigma_{1}$ is the Pauli matrix whose entries represent the graviton and gravitino channels.

Eq. (4.11) has been verified so far at one-loop level, but can be argued to be valid at two loop level, by the following analyticity and unitarity arguments.

First by expanding (4.9) at two loops, we find the elastic amplitude

$$
\frac{A^{(2)}}{s}=F \cdot T \cdot\left[-\frac{2}{3} \delta_{0}^{3}+2 i \delta_{0} \delta_{1}+\delta_{2}+2 i \delta_{0} \delta_{1}^{(N)}\right]
$$

where F.T. indicates the Fourier transform. The latter satisfies analyticity requirements for the following reasons:

(a) The three-body imaginary part related to $\operatorname{Im} \delta_{2}$ is the same as for pure gravity. In fact the gravitino diagrams of fig. 3 lack one factor of $\log s$, with respect to the H-diagram contribution.

(b) The real part is consistent with analyticity. We need to show this fact only for the 
last term in eq. (4.12), the pure gravity part being the same as in ACV II. The phase of the last term is

$$
\delta A^{(2)}=-2 s \delta_{0} \operatorname{Im} \delta_{1}^{(N)}\left[P_{\uparrow \downarrow}+\frac{i}{\pi} \log s\right]
$$

and thus agrees with a combination of the $s$ - $u$ symmetric analytic functions

$$
\begin{gathered}
s^{3}[\log (-s)]^{2}+u^{3}[\log (-u)]^{2} \sim s^{3}\left(\frac{1}{2}+\frac{i}{\pi} \log s\right), \\
s^{3} P_{\uparrow \downarrow}+u^{3} P_{\uparrow \uparrow} \sim s^{3}\left(P_{\uparrow \downarrow}-\frac{1}{2}\right) .
\end{gathered}
$$

Finally, the inelastic term in (4.11) agrees with the explicit evaluation of Appendix B, and makes (4.11) unitary at two loop level.

It is amusing to note that according to Eqs. (4.9) and (4.12) the spin dependent part of the gravitino contributions to the superstring amplitude up to two loops takes the form $(\mathrm{N}=8)$

$$
P_{\uparrow \downarrow} 4 \frac{\Delta}{s}\left(i \delta_{0}^{2}(b)-\frac{2}{3} \delta_{0}^{3}(b)\right), \quad \Delta \equiv \nabla_{b}^{2},
$$

while the SST ( and N=8 Supergravity) kinematical factor has the asymptotic form

$$
K_{c l} \sim\left(1+4 \frac{t}{s} P_{\uparrow \downarrow}\right)\left(\frac{s}{2}\right)^{4}
$$

By replacing $t=-\mathbf{q}^{2}$ by $\Delta$ in impact parameter space we see the result (4.15) is consistent with factorization of the kinematical factor $K_{c l}$ even at two loop level.

The above arguments suggest that the elastic two loop contribution to the eikonal $\delta_{2}$ computed by ACV II is not modified by gravitino contributions. This fact is perhaps not surprising, in view of the semiclassical interpretation of $\operatorname{Re} \delta_{2}$, given in eq. (1.5). The fermionic gravitino field appears not to contribute to the long distance eikonal in the leading $(1 / \hbar)$ limit $R \sim G E \gg \lambda_{P} \sim \sqrt{G \hbar}$.

In conclusion, our SST calculation confirms that massless gravitino terms do contribute to the one-loop quantum corrections to the eikonal, similarly to what happens in the massive scattering case $[13,14]$. On the other hand, we have also argued that no such contribution is present in the "classical" ACV correction $\delta_{2}$, which becomes the most important one when the gravitational radius exceeds the Planck length.

Acknowledgments One of us (A.B.) is grateful to Nordita and Niels Bohr Institute for the warm hospitality. 


\section{Appendix A}

We describe in the following how to compute the trajectory renormalization, in the limit of small transverse momenta, starting from the integral expression given in eq. (3.4). First of all we observe that $\epsilon=0$ and $A \rightarrow 0$ are the regions of interest to obtain $\mathbf{q}^{2}=0$ singularities in four dimension. Therefore we can use the following approximation

$$
\begin{aligned}
I_{\text {pole }}= & \frac{1}{\pi^{2}}\left(\frac{\sigma}{2}\right)^{-1-\mathbf{q}^{2}} \log \sigma \int_{\gamma} \frac{d n}{2 \pi i} \frac{d m}{2 \pi i} \frac{\Gamma(-n) \Gamma(-m)}{\Gamma(n+1) \Gamma(m+1)} \\
& \times \int_{0}^{1} d x B^{-2}\left(-\frac{A^{2}}{8}\right)
\end{aligned}
$$

where $A$ and $B$ are given in eqs. (3.3b) and (3.2b). By defining $t_{1}, t_{2}$ in terms of the loop momenta as in eq. (3.7b), the integration over $x$ is transformed by the Feynman integral representation

$$
\int_{0}^{1} d x \frac{1}{\mathbf{q}^{2} x(1-x)+2 n x+2 m(1-x)}=\int \frac{d^{2} \mathbf{k}}{\pi} \frac{1}{\left(2 n+t_{1}\right)\left(2 m+t_{2}\right)}
$$

and its derivative

$$
\begin{aligned}
\int_{0}^{1} d x \frac{-1}{\left[\mathbf{q}^{2} x(1-x)+2 n x+2 m(1-x)\right]^{2}} \\
\quad=\int \frac{d^{2} \mathbf{k}}{\pi}\left(\frac{\partial}{\partial t_{1}}+\frac{\partial}{\partial t_{2}}\right) \frac{1}{\left(2 n+t_{1}\right)\left(2 m+t_{2}\right)} .
\end{aligned}
$$

Therefore we can reformulate the integral (A.1) in the following way

$$
\begin{aligned}
I_{\text {pole }}=\left(\frac{\sigma}{2}\right)^{-1-\mathbf{q}^{2}} \log \sigma \int \frac{d^{2} \mathbf{k}}{(2 \pi)^{3}}\left(\frac{\partial}{\partial t_{1}}+\frac{\partial}{\partial t_{2}}\right) \int_{\gamma} \frac{d n}{2 \pi i} \frac{d m}{2 \pi i} \frac{\Gamma(-n) \Gamma(-m)}{\Gamma(n+1) \Gamma(m+1)} \\
\quad \times \frac{\left(2 n+2 m+\mathbf{q}^{2}\right)^{2}}{\left(2 n+t_{1}\right)\left(2 m+t_{2}\right)} .
\end{aligned}
$$

We proceed now to integrate along the contour $\gamma$ in the $n$ and $m$ complex plane shown in fig. 4 , by deforming it to the left as usual. We pick up the poles in $2 n=-t_{1}, 2 m=-t_{2}$ because the singular $\mathbf{q}^{2} \rightarrow 0$ behavior arises from the pinching of the contour between them and the poles in $n=0, m=0$. It is straightforward to obtain

$$
I_{\text {pole }}=\left(\frac{\sigma}{2}\right)^{-1-\mathbf{q}^{2}} \log \sigma \int \frac{d^{2} \mathbf{k}}{(2 \pi)^{3}}\left(\frac{\partial}{\partial t_{1}}+\frac{\partial}{\partial t_{2}}\right) \frac{\left(\mathbf{q}^{2}-t_{1}-t_{2}\right)^{2}}{t_{1} t_{2}}
$$

and using the factors in eq. (2.7a) the expression (3.7) follows. 


\section{Appendix B}

In the following we review an S-matrix computation of the leading term of the gravitino box, in the regime of large center of mass energy $s=4 E_{\mathrm{cm}}^{2}$ and fixed momentum transfer $t=-\mathbf{q}^{2}$. For semplicity we consider $N=1$ Supergravity in $D=4$ and use amplitudes in the helicity basis, explicitly given at tree level for gravitons and gravitinos in ref. [12]

$$
S=\delta_{f i}-i(2 \pi)^{4} \delta^{4}\left(p_{1}+p_{2}-p_{3}-p_{4}\right)\left(p_{3}, p_{4} ; p_{1}, p_{2}\right)
$$

$$
\begin{aligned}
\mathrm{A}_{G G}(2,2 ; 2,2) & =8 \pi G \frac{s^{4}}{s t u}, & \mathrm{~A}_{G g}\left(2,2 ; \frac{3}{2}, \frac{3}{2}\right) & =0 \\
\mathrm{~A}_{G G}(2,-2 ; 2,-2) & =8 \pi G \frac{u^{4}}{s t u}, & \mathrm{~A}_{G g}\left(2,-2 ; \frac{3}{2},-\frac{3}{2}\right) & =-8 \pi G \frac{u^{2} \sqrt{t u}}{t s} \\
\mathrm{~A}_{g g}\left(\frac{3}{2}, \frac{3}{2} ; \frac{3}{2}, \frac{3}{2}\right) & =8 \pi G \frac{s^{4}}{s t u}, & \mathrm{~A}_{g g}\left(\frac{3}{2},-\frac{3}{2} ; \frac{3}{2},-\frac{3}{2}\right) & =8 \pi G \frac{u^{4}}{s t u}
\end{aligned}
$$

In particular we notice that crossing simmetry requires for external gravitons the following relation

$$
\mathrm{A}(2,2 ; 2,2 ; s, t, u)=\mathrm{A}(2,-2 ; 2,-2 ; u, t, s)
$$

and that the supergravity kinematical factor, which coincide with the superstring result at tree level, is given for external gravitons by the following expression in the helicity base

$$
K \propto s^{4}\left(P_{\uparrow \uparrow \uparrow \uparrow}+P_{\downarrow \downarrow \downarrow \downarrow}\right)+u^{4}\left(P_{\uparrow \downarrow \uparrow \downarrow}+P_{\downarrow \uparrow \downarrow \uparrow}\right)+t^{4}\left(P_{\uparrow \downarrow \downarrow \uparrow}+P_{\downarrow \uparrow \uparrow \downarrow}\right)
$$

where the $P$ 's are projection operators on external helicity states. In the high energy limit the leading and next to leading terms conserve the helicity of the fast legs and admit the simplified notation

$$
K \simeq s^{4}+4 s^{3} t P_{\uparrow \downarrow}
$$

where $P_{\uparrow \downarrow}=P_{\uparrow \downarrow \uparrow \downarrow}+P_{\downarrow \uparrow \downarrow \uparrow}$.

We compute now the s-channel discontinuity due to gravitinos according to the asymptotic formula

$$
\operatorname{ImA}_{G G}^{+}=\frac{1}{16 \pi^{2} s} \int d^{2} \mathbf{k A}_{G g}^{+}(\mathbf{k}) \mathrm{A}_{G g}^{-}(\mathbf{q}-\mathbf{k})
$$


It is straightforward to prove, using eq. (B.2), that this is proportional to $P_{\uparrow \downarrow}$, precisely

$$
\operatorname{ImA}_{G G}=\frac{1}{16 \pi^{2} s}(8 \pi G)^{2} s^{3} \int \frac{d^{2} \mathbf{k}}{\sqrt{\mathbf{k}^{2}(\mathbf{q}-\mathbf{k})^{2}}} \cong 4 \pi G^{2} s^{2} \log \frac{\Lambda^{2}}{\mathbf{q}^{2}} P_{\uparrow \downarrow} .
$$

The leading real part of this amplitude can be obtained observing that the following $s$ - $u$ symmetric analytic function has the same discontinuity

$$
s^{2} \log (-s) P_{\uparrow \downarrow}+u^{2} \log (-u) P_{\uparrow \uparrow} \simeq s^{2}\left(-i \pi P_{\uparrow \downarrow}+\log s\right)
$$

and the impact parameter transform (4.9) follows for $N$ supergravity.

Finally we remind that the projector in eq. (4.11) operates over gravitons and gravitinos helicity states according to the chosen channel, and gives the following amplitude $\mathrm{A}_{G g}$ for the production of any pair of gravitinos

$$
\mathrm{A}_{G g}=4 s \int d^{2} \mathrm{~b} e^{i \mathrm{~b} \cdot \mathbf{q}} \sqrt{\operatorname{Im} \delta_{1 g}}=\frac{8 \pi G s^{\frac{3}{2}}}{|\mathbf{q}|}
$$

which is easily verified to give the same result as eq. (B.2) in the high energy limit. 


\section{References}

[1] G. 't Hooft, Phys. Lett. B198 (1987) 61; Nucl. Phys. B304 (1988) 867; Nucl. Phys. B335 (1990) 138.

[2] I. Muzinich and M. Soldate, Phys. Rev. D 37 (1988) 353.

[3] D. Amati, M. Ciafaloni and G. Veneziano, Phys. Lett. B197 (1987) 81; Int. J. Mod. Phys. 3A (1988) 1615, hereafter referred to as ACV I.

[4] D. Amati, M. Ciafaloni and G. Veneziano, Nucl. Phys. B347 (1990) 550, hereafter referred to as ACV II.

[5] E. Verlinde and H. Verlinde, Scattering at planckian energies, Princeton preprint PUTP-1279 (1991); see also R.Kallosh, Geometry of scattering at Planckian energies, Stanford preprint SU-ITP 903 (1991).

[6] D. Amati, M. Ciafaloni and G. Veneziano, Planckian scattering beyond the semiclassical approximation, CERN preprint TH.6395/92.

[7] L. N. Lipatov, Phys. Lett B116 (1982) 411; Nucl. Phys. B365 (1991) 614.

[8] B. Sundborg, Nucl. Phys. B306 (1988) 545.

[9] M. Ademollo, A. Bellini and M. Ciafaloni, Phys. Lett. B223 (1989) 318; Nucl. Phys. B338 (1990) 114.

[10] M. B. Green, J. H. Schwarz and E. Witten, Superstring theory (Cambridge University Press, Cambridge, 1987).

[11] A. Bellini, G. Cristofano, M. Fabbrichesi and K. Roland, Nucl. Phys. B356 (1991) 69.

[12] P. Van Nieuwenhuizen, Phys. Rep. 68 (1981) 169; M.T. Grisaru, P. Van Nieuwenhuizen and H.N. Pendleton, Phys. Rev. 15D (1977) 996.

[13] E. Gava, R. Iengo and C.-J. Zhu, Nucl. Phys. B323 (1989) 585.

[14] G. Cristofano, M. Fabbrichesi and K. Roland, Phys. Lett. B246 (1990) 45. 